\title{
Cost-effectiveness of primary prevention of cardiovascular disease with atorvastatin in type 2 diabetes: results from the Collaborative Atorvastatin Diabetes Study (CARDS)
}

\author{
M. Raikou • A. McGuire • H. M. Colhoun • \\ D. J. Betteridge • P. N. Durrington • G. A. Hitman • \\ H. A. W. Neil - S. J. Livingstone • V. Charlton-Menys • \\ J. H. Fuller · CARDS Investigators
}

Received: 5 July 2006 / Accepted: 30 October 2006 / Published online: 31 January 2007

(C) Springer-Verlag 2007

\begin{abstract}
Aims/hypothesis We estimated the cost-effectiveness of atorvastatin treatment in the primary prevention of cardiovascular
\end{abstract}

Electronic supplementary material Supplementary material is available in the online version of this article at http://dx.doi.org/ $10.1007 / \mathrm{s} 00125-006-0561-4$ and is accessible to authorised users.

M. Raikou $(\triangle) \cdot$ A. McGuire

LSE Health and Social Care,

London School of Economics and Political Science,

Houghton Street,

London WC2A 2AE, UK

e-mail: m.raikou@1se.ac.uk

H. M. Colhoun

The Conway Institute, University College,

Dublin, Ireland

D. J. Betteridge

Department of Medicine, The Middlesex Hospital,

University College London,

London, UK

P. N. Durrington $\cdot$ V. Charlton-Menys

Department of Medicine, University of Manchester,

Manchester Royal Infirmary,

Manchester, UK

G. A. Hitman

Centre for Diabetes and Metabolic Disease, Barts and The London, Queen Mary's School of Medicine and Dentistry,

London, UK

H. A. W. Neil

Department of Public Health, University of Oxford,

Oxford, UK

S. J. Livingstone $\cdot$ J. H. Fuller

Department of Epidemiology and Public Health,

Royal Free and University College Medical School,

London, UK disease in patients with type 2 diabetes using data from the Collaborative Atorvastatin Diabetes Study (CARDS).

Subjects and methods A total of 2,838 patients, who were aged 40 to 75 years and had type 2 diabetes without a documented history of cardiovascular disease and without elevated LDL-cholesterol, were recruited from 32 centres in the UK and Ireland and randomly allocated to atorvastatin $10 \mathrm{mg}$ daily $(n=1,428)$ or placebo $(n=1,410)$. These subjects were followed-up for a median period of 3.9 years. Direct treatment costs and effectiveness were analysed to provide estimates of cost per endpoint-free year over the trial period for alternative definitions of endpoint, and of cost per life-year gained and cost per quality-adjusted lifeyear (QALY) gained over a patient's lifetime.

Results Over the trial period, the incremental cost-effectiveness ratio (ICER) was estimated to be $£ 7,608$ per year free of any CARDS primary endpoint; the ICER was calculated to be $£ 4,896$ per year free of any cardiovascular endpoint and $£ 4,120$ per year free of any study endpoint. Over lifetime, the incremental cost per life-year gained was $£ 5,107$ and the cost per QALY was £6,471 (costs and benefits both discounted at 3.5\%).

Conclusions/interpretation Primary prevention of cardiovascular disease with atorvastatin is a cost-effective intervention in patients with type 2 diabetes, with the ICER for this intervention falling within the current acceptance threshold ( $£ 20,000$ per QALY) specified by the National Institute for Health and Clinical Excellence (NICE).

Keywords Cost-effectiveness - Cardiovascular disease . Statins · Type 2 diabetes

\footnotetext{
Abbreviations

CARDS Collaborative Atorvastatin Diabetes Study

CVD cardiovascular disease
} 
ICER incremental cost-effectiveness ratio

NICE National Institute for Health and Clinical Excellence

QALY quality-adjusted life-year

UKPDS United Kingdom Prospective Diabetes Study

\section{Introduction}

Cardiovascular disease (CVD) is a major cause of morbidity and mortality in people with diabetes, who represent approximately $3 \%$ of the UK population and account for between $10 \%$ and $15 \%$ of patients admitted to hospital with myocardial infarction $[1,2]$. At $20 \%$, case fatality is higher than in the non-diabetic population [3]. There is now strong evidence that lipid-lowering therapy is effective in reducing CVD [4-6], but the cost-effectiveness of statins in the primary prevention of CVD in people with diabetes has not been assessed adequately.

The Collaborative Atorvastatin Diabetes Study (CARDS) recently established that treatment of type 2 diabetic patients with atorvastatin (10 $\mathrm{mg}$ daily) is effective in the primary prevention of CVD [7], with a $36 \%$ reduction in CHD events and a $48 \%$ reduction in stroke over the trial period. The economic implications of these results in terms of healthcare expenditure require investigation. This paper reports on the cost-effectiveness of atorvastatin in the primary prevention of CVD using the CARDS data over the duration of the trial and a patient's lifetime.

\section{Subjects and methods}

Trial design and population The design of CARDS, the baseline characteristics of the patient population and the main clinical outcomes have been published previously [7]. The study was a randomised controlled trial designed to assess the effectiveness of atorvastatin $10 \mathrm{mg}$ daily in the primary prevention of major cardiovascular events in patients with type 2 diabetes and without elevated levels of LDL-cholesterol. The trial population consisted of 2,838 patients with type 2 diabetes and no documented prior history of CVD, but with at least one of the following CVD risk factors: retinopathy, micro- or macroalbuminuria, current smoking or hypertension. Eligible patients had an LDL-cholesterol $\leq 4.14 \mathrm{mmol} / 1(160 \mathrm{mg} / \mathrm{dl})$ and a fasting triacylglycerol $\leq 6.78 \mathrm{mmol} / 1(600 \mathrm{mg} / \mathrm{dl})$. The distribution of age, sex and ethnicity, duration of diabetes, $\mathrm{HbA}_{1 \mathrm{c}}$ levels and history of retinopathy, hypertension and existing treatment was the same in both treatment arms at baseline. The mean age of the CARDS population was 62 years, with the majority of patients being white (94\%) and with men predominating (68\%). The median length of follow-up was 3.91 years in the placebo and 3.94 years in the actively treated patients. The primary endpoint in the trial was defined as time to one of the following events: acute CHD (myocardial infarction including silent infarction, unstable angina, acute CHD death), coronary revascularisation procedure, resuscitated cardiac arrest or stroke [7].

Economic perspective The analysis in this paper assesses the cost-effectiveness of the intervention from the perspective of a healthcare purchaser as represented by the UK National Health Service. Only the direct costs to the health service are therefore taken into account. Two time horizons are considered in this study. The first corresponds to the duration of the CARDS trial, and consequently the incremental costeffectiveness ratio (ICER) is based on the within-trial effectiveness. The second extends to a patient's lifetime, and the cost-effectiveness of the intervention is expressed as incremental cost per life-year gained and incremental cost per quality-adjusted life-year (QALY) gained.

Endpoints The within-trial ICER was calculated for three definitions of clinical endpoints following those used in the CARDS study. The first, referred to as any primary endpoint, is a composite measure that comprises fatal and non-fatal myocardial infarction, silent myocardial infarction, CHD death, unstable angina, coronary artery revascularisation, resuscitated cardiac arrest and fatal and non-fatal stroke (i.e. major CVD events). A broader secondary endpoint was defined as 'any cardiovascular endpoint' and it encompassed any major CVD event, as well as angina, requiring acute hospital attendance, other acute CHD events, non-fatal transient ischaemic attack, peripheral vascular disease requiring hospitalisation and other acute hospitalised non-fatal cardiovascular events (i.e. all CVD events). A third endpoint definition comprised all study endpoints recorded within the CARDS trial (i.e. any CVD event and death from any cause). The measure of effectiveness was thus defined as the time that a patient was free of the endpoint under study. The ICER was calculated for the within-trial population as the incremental cost per event-free time for the three composite endpoints defined above and for the lifetime extrapolation as both incremental cost per life-year gained and QALY gained.

Resource consumption and costs Data were collected for each patient in the study, recording the dosage and the start and end date of the trial drug (on the atorvastatin arm) and the use of any additional statin therapy for both treatment arms, as well as the number of clinic visits, the tests performed at these visits and the hospitalisations for endpoints. The dates of admission and discharge were recorded for all hospitalisations, and a study endpoint was 
assigned to each hospitalisation providing the main reason for admission. Dates of admission and discharge relating to hospitalisation for serious adverse events were not recorded in the trial owing to data collection constraints. However, as serious adverse events only affected approximately $1 \%$ of patients in each arm and the incidence and case-mix of serious adverse events were similar for the two trial arms, serious adverse events were excluded from the analysis.

Unit cost information was combined with individual resource volumes to calculate the direct treatment cost per patient over the study period. Various sources provided data on the unit costs, all of which are reported in 2003/2004 prices in pounds sterling (mean exchange rate GBP/Euro on 1 January 2004: $€ 1.43$ to $£ 1.00$ ). Drug unit cost information was obtained from the British National Formulary [8]. Clinic visit and laboratory test unit costs were based on the Personal and Social Services Research Unit costing of health and social care [9]. Hospital care unit costs were based on the UK Department of Health hospital tariffs [10] converted into per day costs for each of the hospital episodes recorded in the study. These were then combined with the individual patient length of hospital stay to derive the total cost of inpatient stay for each patient (see Electronic supplementary material [ESM] Table 1). All costs were discounted at the $3.5 \%$ per year rate as recommended by the National Institute for Health and Clinical Excellence (NICE) [11], and a mean cost per patient over the study period was calculated for each arm of the study.

Lifetime extrapolation Given the chronic nature of type 2 diabetes, an analysis of cost and effectiveness over lifetime was undertaken to evaluate the long-term use of atorvastatin therapy. In this analysis, the results from the CARDS trial were extrapolated to estimate the incremental cost per life-year gained and the incremental cost per QALY gained with atorvastatin $10 \mathrm{mg}$ over a patient's lifetime. The main assumption was that treatment with atorvastatin continued over the duration of a patient's life, with an associated continuing treatment benefit represented by the difference in cumulative survival between the two arms observed at the end of the CARDS trial. It was further assumed that if an individual from the placebo population experienced a non-fatal CVD event, he or she was switched to atorvastatin therapy for the duration of their remaining lifetime.

A non-parametric approach was adopted in the extrapolation of the CARDS results beyond the end of the trial period using life tables. Alternative parametric models were also considered, but the application of this method was unsuccessful because of the small number of fatal events observed over the trial period in both arms of the trial. Thus, the calculation of future life-year gains was based on the assumption of continuing treatment with atorvastatin and maintained treatment benefit beyond the trial end applied to the mortality rates of a UK population of patients with type 2 diabetes drawn from a recent longitudinal study [12]. Comparison of the age- and sex-specific cumulative hazard rates for the CARDS placebo population and this UK longitudinal study of patients with type 2 diabetes showed no significant difference. Thus, lifetime cumulative mortality for the placebo population was estimated from the derived life expectancy in this longitudinal analysis of a type 2 diabetic population in the United Kingdom [12]. The lifetime atorvastatin cumulative mortality was based on the assumption that the therapeutic benefit observed at the end of the CARDS study was maintained over a patient's lifetime. The assumption of continuing on-treatment benefit is justified given the acknowledged long-term benefits of statin therapy with continued treatment [13]. Furthermore, given the early termination of the trial, this is likely to be a conservative estimate of treatment benefit. The gain in life-years was then estimated as the difference in the area under the extrapolated cumulative survival curves between the two groups.

This may be regarded as a conservative estimate, as it models the gain in life expectancy arising solely from differences in fatal events without taking account of the impact of non-fatal events on subsequent mortality. That is, differences in life expectancy may be attributed to atorvastatin, as this treatment is also associated with lower rates of nonfatal CVD events, which are expected to lead to lower subsequent mortality. The impact of non-fatal events on subsequent mortality was recognised by the United Kingdom Prospective Diabetes Study (UKPDS) investigators and was modelled explicitly in their extrapolation of the UKPDS trial results over a patient's lifetime [14]. In the sensitivity analysis in this study, a further supplementary assumption was made, namely that non-fatal CVD events were associated with an increase in the annual probability of mortality in the same manner and using the same calculations adopted by the UKPDS investigators [14]. To undertake this part of the analysis, it was necessary to extrapolate non-fatal CVD event rates beyond the trial end. This was undertaken on the assumption that subsequent event rates were equal to those observed in the final year of trial follow-up and that this difference was maintained over time.

QALYs Quality-of-life assessment was based on estimated differences in quality-of-life tariffs owing to differences in non-fatal CVD events. Each non-fatal CVD endpoint was assumed to be associated with a specific decrease in a patient's quality of life. The value of this decrease was obtained from the analysis of the quality-of-life scores of patients with type 2 diabetes by the UKPDS investigators (UKPDS 62) [15]. The mean European Quality of Life 5dimensions (EQ-5D) tariff score for their diabetic population was $0.77(\mathrm{SD}=0.27)$. The incremental change in this aggregate value was calculated for myocardial infarction, 


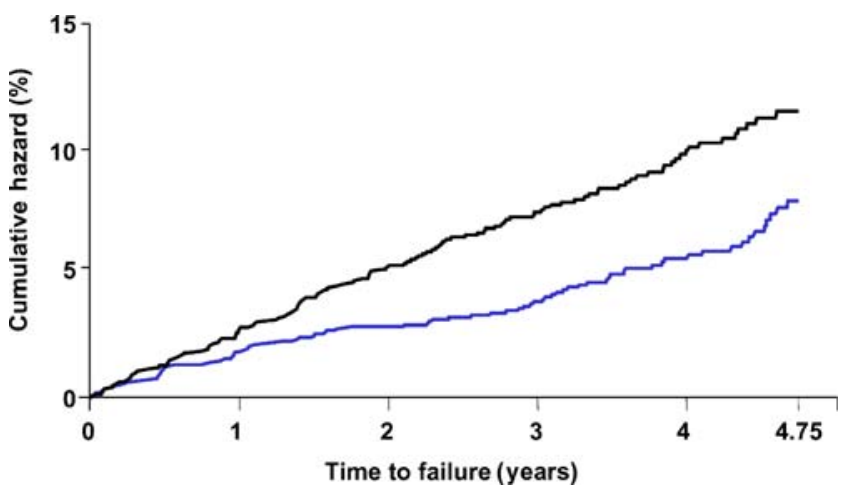

Fig. 1 Nelson-Aalen cumulative hazard estimates for within-trial time to primary endpoint. Black line, placebo; blue line, atorvastatin

ischaemic heart disease and stroke as -0.055 (95\% CI. $-0.067,-0.042),-0.09$ (95\% CI. $-0.126,-0.054)$ and -0.164 (95\% CI. $-0.222,-0.105)$, respectively. QALYs gained in this analysis were calculated applying these incremental changes in quality-of-life scores to the relevant non-fatal CVD events reported by CARDS. This leads to an estimate of changes in quality-of-life scores (from the reference case of 0.77 ) in each of the trial arms directly relating to the observed non-fatal CVD events, which in turn, combined with estimated life expectancy in each treatment arm, allows calculation of the gain in QALYs attributable to atorvastatin.

Calculation of the associated cost is based on the assumption of continuing atorvastatin therapy over the patient's lifetime as described previously. If patients in the placebo arm had a CVD event, they were switched to statin therapy and this continued over their remaining lifetime, while the cost of a non-fatal CVD event for both the treatment and the placebo population was estimated as a weighted average with the weights reflecting the proportions of different types of CVD events occurring within the trial period. Indeed data on the within-trial population showed that a small number of patients in the placebo population, fewer than $10 \%$, were administered a statin during the trial period.

Sensitivity analysis As mentioned previously in the section on lifetime extrapolation, part of the sensitivity analysis addressed the impact of non-fatal CVD events on the probability of subsequent mortality. Further sensitivity analysis was undertaken in the extrapolation. First, one-way parameter sensitivity analysis was performed on the unit cost of atorvastatin, assuming a $15 \%$ decrease in the unit cost of atorvastatin - a change that would move the statin under study towards a generic statin price level. Secondly, a different comparator treatment was considered by decreasing the event rates observed within the placebo population in such a manner that the level of effectiveness became similar to the level of effectiveness of a generic statin population for the placebo population. In addition, probabilistic sensitivity analysis was undertaken to address the uncertainty associated with the survival probabilities used in the extrapolation of the cumulative survival curves. For each year beyond the trial end, the cumulative survival probability for the atorvastatin arm was assumed to be drawn from an underlying (uniform) distribution bounded by the cumulative survival probabilities for the previous and the next years of extrapolation. This change in the probability values results in different estimates for the cost of atorvastatin and for the incremental cost per QALY. The process of changing the probability values was repeated 1,000 times and the empirical distribution of the resultant ICERs is reported in the results section. A final analysis calculated within-trial and extrapolated ICERs for high-, medium- and low-risk populations. The UKPDS risk engine was applied to the CARDS population to predict the probability of a CHD event for each CARDS patient, and the findings were then used to assign CARDS subjects to a risk tertile. The resultant ICER was then calculated for each of these risk tertiles.

Table 1 Kaplan-Meier estimates of mean time to event for the within-study period for various study endpoints

\begin{tabular}{llllll}
\hline $\begin{array}{l}\text { Definition of } \\
\text { endpoint }\end{array}$ & & $\begin{array}{l}\text { Placebo } \\
\text { (undiscounted) }\end{array}$ & $\begin{array}{l}\text { Atorvastatin } \\
\text { (undiscounted) }\end{array}$ & $\begin{array}{l}\text { Difference in treatment } \\
\text { effect (effects } \\
\text { undiscounted) }\end{array}$ & $\begin{array}{l}\text { Difference in treatment } \\
\text { effect (effects } \\
\text { discounted at 3.5\%) }\end{array}$ \\
\hline Primary endpoint & $\begin{array}{l}\text { Mean time to event (years) } \\
\text { Number of events }\end{array}$ & $\begin{array}{l}5.19 \\
127\end{array}$ & $\begin{array}{l}5.28 \\
83\end{array}$ & 0.0875 \\
Any cardiovascular \\
endpoint & Mean time to event (years) & 4.99 & 5.13 & 44 \\
& Number of events & 189 & 134 & 0.136 \\
All-cause mortality & Mean time to event (years) & 5.33 & 5.35 & 55 \\
& Number of events & 82 & 61 & 0.011 \\
Any study endpoint & Mean time to event (years) & 4.89 & 5.053 & 21 \\
& Number of events & 229 & 167 & 0.159 \\
\hline
\end{tabular}


Table 2 Incremental cost-effectiveness ratios for the major endpoint categories over the trial period

\begin{tabular}{|c|c|c|c|c|c|c|c|}
\hline $\begin{array}{l}\text { Definition of } \\
\text { endpoint }\end{array}$ & $\begin{array}{l}\text { Placebo total } \\
\text { cost }(£)\end{array}$ & $\begin{array}{l}\text { Placebo } \\
\text { effect }\end{array}$ & $\begin{array}{l}\text { Atorvastatin } \\
\text { Total cost }(£)\end{array}$ & $\begin{array}{l}\text { Atorvastatin } \\
\text { effect }\end{array}$ & $\begin{array}{l}\text { Difference in } \\
\text { costs }(£)\end{array}$ & $\begin{array}{l}\text { Difference in } \\
\text { effects }\end{array}$ & ICER (£) \\
\hline Primary endpoint & $1,003.746$ & 4.391 & $1,524.982$ & 4.460 & 521.236 & 0.068 & $\begin{array}{l}\text { 7,608.39 per primary } \\
\text { endpoint-free year }\end{array}$ \\
\hline $\begin{array}{l}\text { Any cardiovascular } \\
\text { endpoint }\end{array}$ & & 4.235 & & 4.342 & & 0.106 & $\begin{array}{l}\text { 4,896.48 per cardiovascular } \\
\text { endpoint-free year }\end{array}$ \\
\hline $\begin{array}{l}\text { Any study } \\
\text { endpoint }\end{array}$ & & 4.153 & & 4.280 & & 0.126 & $\begin{array}{l}4,119.85 \text { per year free of any } \\
\text { endpoint }\end{array}$ \\
\hline
\end{tabular}

Incremental cost-effectiveness ratio $(I C E R)$ is the cost per event-free time gained (in $£, 2003 / 2004$ prices, costs and effects discounted at $3.5 \%$ )

Ethical approval The study was conducted in accordance with the Declaration of Helsinki and the Guidelines on Good Clinical Practice. Each centre obtained Local Research Ethics Committee approval following approval from the Multicentre Research Ethics Committee. All patients gave fully informed written consent.

\section{Results}

Within-trial Figure 1 displays the within-trial cumulative hazard curves for both arms of the trial for the primary endpoint in the CARDS trial. Table 1 shows the estimates of effectiveness used in the calculation of the within-trial period ICERs. The table also reports the total number of endpoints for each of the definitions. The difference in the mean time to event between the two arms of the study (discounted at $3.5 \%$ per year) forms the basis of the effectiveness measure. Thus, it can be seen that after discounting benefits, the within-trial gain in effectiveness ranges between 0.0078 and 0.126 years for the broadest endpoint definition.

Over the total study period, the difference in average discounted treatment cost is $£ 521$ per patient (ESM Table 2). Table 2 presents the within-trial calculated ICERs for all definitions of endpoints. Based on the within-trial analysis, it costs $£ 7,608$ on average to gain a year of life free from any primary endpoint. The ICER is $£ 4,896$ per CVD endpoint-free year and $£ 4,120$ per any trial endpoint-free year. In addition, the estimated cost per CARDS primary endpoint averted is $£ 16,916$. Confidence intervals for the ICER were calculated using Fieller's method [16], while the cost-effectiveness acceptability curve for the primary trial endpoint is reported in Fig. 2 and shows the probability that the intervention is cost-effective for different ceiling values of ICER. Based on the calculated ICER relating to any primary trial endpoint, there is a $50 \%$ likelihood that the cost per primary endpoint-free year is below $£ 7,608$ and a $90 \%$ likelihood that it is below $£ 9,000$.

\section{Lifetime}

Table 3 reports the extrapolated results on life expectancy. As can be seen, life expectancy was estimated to be 17.29 years (undiscounted) and 12.48 years (discounted) (at $3.5 \%$ per annum) on average in the placebo population and 18.20 years (undiscounted) and 12.97 years (discounted) in the atorvastatin population, resulting in a gain of 0.49 years (discounted) attributable to atorvastatin therapy over a patient's lifetime. The ICER was $£ 5,107$ per life-year gained with costs and effects discounted at $3.5 \%$, and the incremental cost per QALY was $£ 6,471$ with costs and effects discounted at $3.5 \%$. The treatment costs are dominated by the cost of atorvastatin, which is calculated to be $£ 3,117$ per patient over lifetime. The second most important cost category is the cost of drug therapy for those on placebo who are assumed to switch to atorvastatin following a CVD event ( $£ 162$ per patient over lifetime discounted at $3.5 \%$ ).

Sensitivity analysis Assuming a 15\% decrease in the unit cost of atorvastatin, which brings the price of atorvastatin closer to a generic statin price, the resultant cost per primary endpoint-free year fell to $£ 5,928$ (the ICER was $£ 3,831$ per any CVD endpoint-free year and $£ 3,223$ per any study endpoint-free year), while the cost per life-year gained fell to $£ 4,203$ and the cost per QALY fell to $£ 5,325$ (discounting

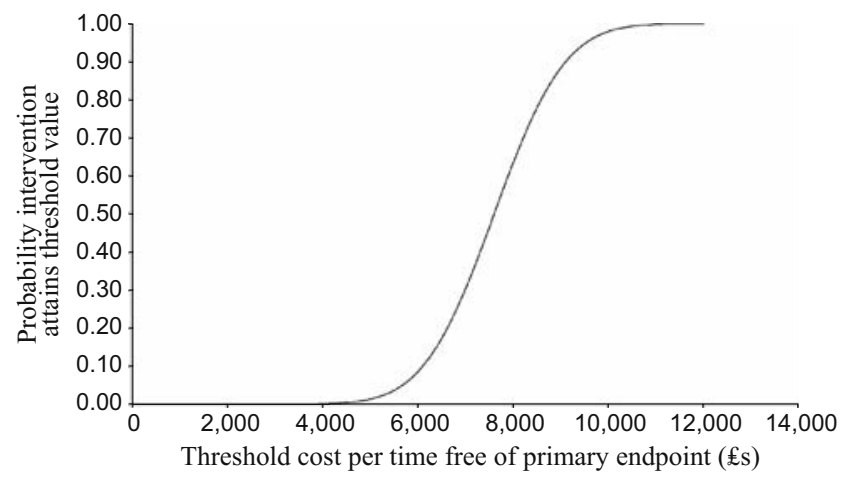

Fig. 2 Within-trial cost-effectiveness acceptability curve 
Table 3 Incremental cost-effectiveness ratios for cost per life-year gained and cost per QALY

\begin{tabular}{lll}
\hline & Placebo (mean) & Atorvastatin (mean) \\
\hline $\begin{array}{l}\text { Life-years (undiscounted) } \\
\text { Life-year gained (undiscounted) }\end{array}$ & 17.29 & 18.20 \\
$\begin{array}{l}\text { Life-years (discounted at 3.5\%) } \\
\text { Life-year gained (discounted at 3.5\%) }\end{array}$ & 12.48 & 0.9091 \\
QALYs (undiscounted) & 13.28 & 0.9790 \\
QALYs (discounted at 3.5\%) & 9.59 & 13.99 \\
QALYs gained (discounted at 3.5\%) & & 9.97 \\
Incremental costs per life-year gained (£) & 2,755 (undiscounted life-year gained) & 0.3871 \\
Incremental costs per QALY (£) & 3,513 (undiscounted QALYs) & 6,107 (discounted life-year gained) \\
Incremental costs per life-year gained (assuming & 2,760 (undiscounted life-year gained) & 5,116 (discounted life-year gained) \\
$\quad$ fatal events as proportion of non-fatal) (£) & & 6,848 (discounted QALYs) \\
Incremental costs per QALY (assuming fatal & 3,520 (undiscounted QALYs) & \\
events as proportion of non-fatal) (£) & &
\end{tabular}

both costs and effects by 3.5\%). Sensitivity analysis also assessed the impact of a first non-fatal CVD event on subsequent mortality. Using the annual probability value of a fatal CHD and a fatal stroke event following a first non-fatal event of 0.004 reported by the UKPDS investigators [14], the additional gain in life expectancy attributable to treatment with atorvastatin was assessed and the resulting ICERs are also reported in Table 3. The cost per life-year gained is now $£ 5,116$ and the cost per QALY is $£ 6,848$ (both costs and effects discounted at $3.5 \%$ per year).

Results relating to lifetime cost-effectiveness were based on extrapolation of within-trial results by matching individuals by age and sex to estimate average population effects. This extrapolation did not yield individual patient costs, survival or QALYs, and therefore uncertainty in the estimates cannot be assessed by means of confidence intervals. Statistical inference cannot be applied directly to these calculations. Instead, uncertainty was address by introducing randomness into the estimates using simulation. This resulted in an empirical distribution of the incremental cost per QALY, as presented in Fig. 3, based on a simulation of 1,000 replications of the average cumulative survival probability. The resultant ICER ranged from

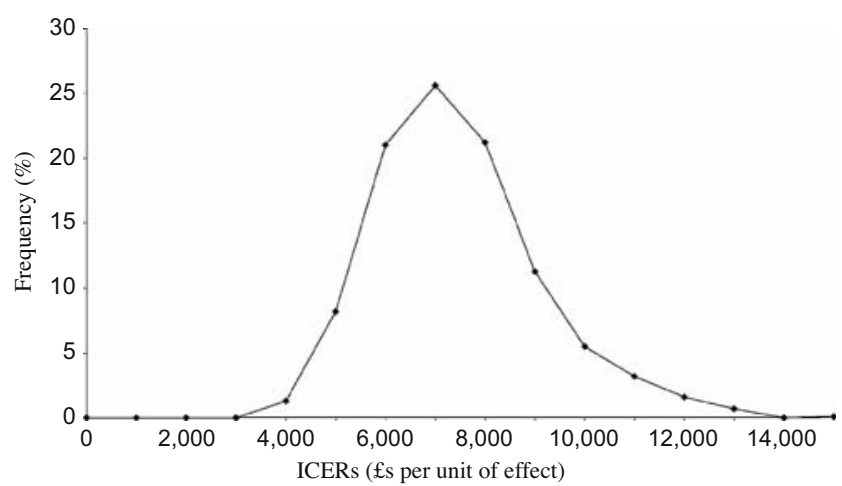

Fig. 3 Incremental cost-effectiveness ratios (ICERs) from probability sensitivity analysis
$£ 3,321$ to $£ 14,547$ with most of the values lying between $£ 6,000$ and $£ 8,000$.

Sensitivity analysis was also used to investigate the incremental cost-effectiveness of atorvastatin compared with a lower-dose statin priced at $10 \%$ of the unit cost of atorvastatin. In the CARDS trial, a relative risk reduction of $32 \%$ on all CVD events was observed with atorvastatin. If the findings of Pedersen et al. [17] are transferable to the CARDS population, a $16 \%$ relative risk reduction, reflecting high- and low-dose use, may be assumed. Applying this relative risk reduction to the CARDS cumulative mortality rates, the resultant cost per QALY is $£ 10,568$ (both costs and effects discounted at $3.5 \%$ ).

Tables 4 and 5 report the within-trial and extrapolated ICERs when the CARDS population was classified according to predicted risk of heart disease into high-, mediumand low-risk groups. The risk populations were defined by allocating the CARDS population to risk tertiles with the help of the UKPDS risk engine [18]. Individual patient characteristics from the CARDS trial population were used to populate the UKPDS risk engine and the estimated 5.5year risk of a CHD was calculated. In applying the UKPDS risk model for CHD, which is an incidence model, adjustment was made to the CARDS patients' ages to reflect their age at the point of diagnosis with type 2 diabetes. Use of the UKPDS model to identify risk tertiles is justified in that the modelled survival probabilities fall within the $95 \%$ non-parametric confidence intervals of the rates observed within the CARDS study for the relevant population endpoints. Indeed, there was a slight overprediction of the survival rates for the placebo population and a slight under-prediction of these rates for the atorvastatin group, indicating that use of the UKPDS model in this manner may have led to conservative estimates. The estimated probabilities formed the basis of a risk distribution, which was then divided into tertiles. The resultant ICERs for the within-trial analysis (Table 4) lie between 
Table 4 Within trial incremental cost-effectiveness ratio for cost per cardiovascular event-free year for risk categories defined by UK Prospective Diabetes Study risk engine

\begin{tabular}{llll}
\hline Risk category & Cost difference $(£)$ & Effect difference & Incremental cost-effectiveness ratio $(£)$ \\
\hline Low risk & 655 & 0.109 & 5,983 per event-free year \\
Medium risk & 564 & 0.164 & 3,436 per event-free year \\
High risk & 540 & 0.260 & 2,077 per event-free year \\
\hline
\end{tabular}

Values (costs in $£, 2003 / 2004$ prices) are by tertiles

$£ 2,077$ per CVD endpoint-free year for the high-risk group and $£ 5,983$ per CVD endpoint-free year for the low-risk group. The ICERs for the lifetime analysis (Table 5) are calculated to be $£ 4,001$ per QALY for the high-risk group, $£ 8,523$ for the medium-risk group and $£ 20,781$ for the lowrisk group.

\section{Discussion}

The recognition that patients with diabetes have a higher risk of developing CVD than the general population is reflected in current UK guidelines including those from the National Service Framework [19] and the Joint British Recommendations [20]. The latest evidence suggests that most patients with diabetes, even those without raised cholesterol or established CVD, would benefit from statin treatment $[21,22]$. Even though prescription of statins has increased since the introduction of national guidelines, statin use in the United Kingdom appears to lag behind that of many parts of Europe and the United States [23]; therefore, many patients are denied effective treatment. This may be explained in part by a lack of information on the economic consequences of statin treatments in both the short and longer term, particularly in primary prevention. This analysis supports the use of atorvastatin in the primary prevention of CVD in patients with type 2 diabetes by showing that this intervention, apart from being clinically effective, is also cost-effective under a range of assumptions. The calculated ICERs fall within the NICE costeffectiveness threshold of $£ 20,000$ per QALY, and on this basis, treatment with atorvastatin in the primary prevention of CVD in patients with type 2 diabetes favourably compares with competing healthcare interventions [11].

The results of this analysis need to be interpreted with some caution because of the following limitations. First, no account was taken of co-medications other than concomitant statin use. This might be considered an important omission given the high level of co-medication in the CARDS diabetic population. However, the proportion of patients on approximately 20 major categories of comedication showed no statistical difference in usage between the two arms of the trial. For instance, similar proportions of patients in the two arms of the trial were on oral hypoglycaemic medication (65\%), insulin (14\%) and antihypertensive agents (84\%). Secondly, no account was taken of the degree of compliance. Nor was any account taken of serious adverse events, the rate of which was low and similar in both arms (1.1\%). Despite these limitations of the analysis, the reported results are most probably conservative, given the assumptions underlying the extrapolation, and the results of the sensitivity analyses.

The within-trial analysis reported by the Heart Protection Study investigators suggests that statin therapy is costeffective across a wide range of individuals [24]. The present study supports this finding by using the evidence gained from CARDS to assess the cost-effectiveness of atorvastatin therapy in the primary prevention of CVD in a patient population with type 2 diabetes. CARDS therefore makes an important contribution to the clinical and economic evidence with regard to the primary prevention of CVD in patients with type 2 diabetes, which may aid the NICE technology appraisal of the use of statins in coronary

Table 5 Incremental cost-effectiveness ratio for cost per life-year gained and cost per QALY gained for risk categories defined by UK Prospective Diabetes Study risk engine

\begin{tabular}{llllll}
\hline $\begin{array}{l}\text { Risk } \\
\text { category }\end{array}$ & $\begin{array}{l}\text { Cost } \\
\text { difference }(£)\end{array}$ & $\begin{array}{l}\text { Effect } \\
\text { difference, LYG }\end{array}$ & $\begin{array}{l}\text { Effect difference, } \\
\text { QALY }\end{array}$ & $\begin{array}{l}\text { Incremental cost-effectiveness } \\
\text { ratio per LYG }(£)\end{array}$ & $\begin{array}{l}\text { Incremental cost-effectiveness } \\
\text { ratio per QALY (£) }\end{array}$ \\
\hline Low risk & 2,805 & 0.161 & 0.135 & 17,424 & 20,781 \\
Medium risk & 2,719 & 0.401 & 0.319 & 6,781 & 8,523 \\
High risk & 2,574 & 0.824 & 0.644 & 3,124 & 4,001 \\
\hline
\end{tabular}

Values (costs in $£, 2003 / 2004$ prices) are by tertiles. Costs and LYG/QALYs discounted at $3.5 \%$ per annum, rounding errors present $L Y G$, life-year gained 
heart disease. This finding holds even when a low-risk population is considered. In line therefore with current UK recommendations and given the proven clinical benefits and the ICERs reported here, primary prevention of CVD with atorvastatin is justified as a standard intervention in patients with type 2 diabetes.

Acknowledgements A non-restricted grant was provided by Pfizer Inc. to A. McGuire and M. Raikou to enable this research. The health economic analysis was undertaken by M. Raikou and A. McGuire. All authors contributed towards interpretation of the data and revision of the manuscript and have seen and approved the final version.

Duality of interest D. J. Betteridge and H. M. Colhoun have served as consultants to and received travel expenses and payment for speaking at meetings from Pfizer. P. N. Durrington has received travel expenses, payment for speaking at meetings and funding for research from Pfizer. J. H. Fuller and G. A. Hitman have served as consultants to and received travel expenses, payment for speaking at meetings or funding for research from pharmaceutical companies marketing lipidlowering drugs, including AstraZeneca and Pfizer. A. J. McGuire has served as a consultant to and received travel expenses and funding for research from pharmaceutical companies marketing lipid-lowering drugs including AstraZeneca, Merck Sharp and Dohme, and Pfizer. H. A. W. Neil has served as consultant to and received travel expenses, payment for speaking at meetings or funding for research from pharmaceutical companies marketing lipid-lowering drugs, including AstraZeneca, Merck Sharp and Dohme and Pfizer. M. Raikou has served as a consultant to and received travel expenses and funding for research from Pfizer. The University College London coordinating centre was partly funded by a grant from Pfizer UK and Pfizer Inc. to University College London. S. J. Livingstone has no conflicts of interest to declare.

\section{References}

1. British Medical Association (2004) Diabetes mellitus an update for healthcare professionals. BMA, London

2. Barnett AH, O'Gara G (2004) Churchill's in clinical practice series: diabetes and the heart. Churchill Livingstone, Oxford

3. Singer DE, Moulton AW, Nathan DM (1989) Diabetic myocardial infarction. Interaction of diabetes with other preinfarction risk factors. Diabetes 38:350-357

4. Banerji MA (2005) Statins and the prevention of stroke in diabetes. Curr Diab Rep 5:1-3

5. Pimpinella G, Bertini Malgarini R, Martini N (2004) Statins for patients with type 2 diabetes. Lancet 364:1933

6. Gami AS, Montori VM, Erwin PJ, Khan MA, Smith SA; Evidence in Diabetes Enquiry System (EVIDENS) Research Group (2003) Systematic review of lipid lowering for primary prevention of coronary heart disease in diabetes. BMJ 326:528-529

7. Colhoun HM, Betteridge DJ, Durrington PN et al; CARDS investigators (2004) Primary prevention of cardiovascular disease with atorvastatin in type 2 diabetes in the Collaborative Atorvastatin Diabetes Study (CARDS): multicentre randomised placebo-controlled trial. Lancet 364:685-696
8. British National Formulary (2004) BMA and RPSUK, London

9. Curtis L, Netten A (2004) Unit costs of health and social care. PSSRU, University of Kent, Canterbury

10. Department of Health (2005) Reference costs 2004. Available from http://www.dh.gov.uk/PublicationsAndStatistics/Publications/ PublicationsPolicyAndGuidance/PublicationsPolicyAndGuidance Article/fs/en?CONTENT_ID $=4105545 \& \mathrm{chk}=$ znAfqu, last accessed 19 September 2006

11. National Institute for Clinical Excellence (2004) Guide to the methods of technology appraisal (reference 0515)

12. Roper N, Bilous R, Kelly W, Unwin NC, Connelly VM (2001) Excess mortality in a population with diabetes and the impact of material deprivation: longitudinal, population-based study. BMJ 322:1389-1393

13. Strandberg TE, Pyörälä K, Cook TJ et al (2004) Mortality and incidence of cancer during 10-year follow-up of the Scandinavian Simvastatin Survival Study (4S). Lancet 364:771-777

14. Clarke PM, Gray AM, Briggs A et al; UK Prospective Diabetes Study (UKPDS) Group (2004) A model to estimate the lifetime health outcomes of patients with type 2 diabetes: the United Kingdom Prospective Diabetes Study (UKPDS) outcomes model (UKPDS no. 68). Diabetologia 47:1747-1759

15. Clarke P, Gray A, Holman R (2002) Estimating utility values for health states of type 2 diabetic patients using the EQ-5D (UKPDS 62). Med Decis Mak 22:340-349

16. Fieller EC (1954) Some problems in interval estimation. J R Stat Soc Ser B 16:175-185

17. Pedersen TR, Faergeman O, Kastelein JJP et al; Incremental Decrease in End Points Through Aggressive Lipid Lowering (IDEAL) Study Group (2005) High-dose atorvastatin vs usualdose simvastatin for secondary prevention after myocardial infarction: the IDEAL study: a randomized controlled trial. JAMA 294:2437-2445

18. Stevens R, Kothari V, Adler AI, Stratton IM; United Kingdom Prospective Diabetes Study (UKPDS) Group (2001) The UKPDS risk engine: a model for the risk of coronary heart disease in type 2 diabetes (UKPDS 56). Clin Sci (Lond) 101:671-679

19. Department of Health (2000) Coronary heart disease: national service framework for coronary heart disease - modern standards and service models. Department of Health, London

20. No authors given (1998) Joint British recommendations on prevention of coronary heart disease in clinical practice. British Cardiac Society, British Hyperlipidaemia Association, British Hypertension Society, endorsed by the British Diabetic Association. Heart 80:S1-S29

21. Heart Protection Study Collaborative Group (2002) MRC/BHF Heart Protection Study of cholesterol lowering with simvastatin in 20,536 high-risk individuals: a randomised placebo-controlled trial. Lancet 360:7-22

22. Sever PS, Dahlof B, Poulter NR et al; ASCOT investigators (2003) Prevention of coronary and stroke events with atorvastatin in hypertensive patients who have average or lower-thanaverage cholesterol concentrations, in the Anglo-Scandinavian Cardiac Outcomes Trial-Lipid Lowering Arm (ASCOT-LLA): a multicentre randomised controlled trial. Lancet 361:11491158

23. Cowie M, Betteridge J, Hobbs R et al (2003) Lipid lowering. Why does the UK lag behind? Update 14 August 2003, pp 226230

24. Mihaylova B, Briggs A, Armitage J, Parish S, Gray A, Collins R; Heart Protection Study Collaborative Group (2005) Cost-effectiveness of simvastatin in people at different levels of vascular disease risk: economic analysis of a randomised trial in 20,536 individuals. Lancet 365:1779-1785 